\title{
APPLICATION OF LAKSHMIKANTHAM'S MONOTONE-ITERATIVE TECHNIQUE TO THE SOLUTION OF THE INITIAL VALUE PROBLEM FOR IMPULSIVE INTEGRO-DIFFERENTIAL EQUATIONS ${ }^{1}$
}

\author{
D.D. BAINOV and S.G. HRISTOVA ${ }^{2}$ \\ Plovdiv University \\ Department of Mathematics \\ Plovdiv, BULGARIA
}

\begin{abstract}
In the present paper, a technique of V. Lakshmikantham is applied to approximate finding of extremal quasisolutions of an initial value problem for a system of impulsive integro-differential equations of Volterra type.
\end{abstract}

Key words: Monotone-iterative technique, impulsive integrodifferential equations.

AMS (MOS) subject classifications: $\quad 34 \mathrm{~A} 37$.

\section{INTRODUCTION}

The monotone-iterative technique of $\mathrm{V}$. Lakshmikantham is one of the most effective methods for finding approximate solutions of initial value and periodic problems for differential equations. This technique is a fruitful combination of the method of upper and lower solutions and a suitably chosen monotone method [1]-[8].

In the present paper, by means of this monotone-iterative technique, minimal and maximal quasisolutions of the initial value problem for a system of impulsive integrodifferential equations of Volterra type are obtained.

\section{STATEMENT OF THE PROBLEM, PRELIMINARY NOTES}

Consider the initial value problem for the system of impulsive integro-differential equations

\footnotetext{
${ }^{1}$ Received: September, 1992. Revised: February, 1993.

${ }^{2}$ The present investigation is supported by the Ministry of Education and Science of the Republic of Bulgaria under Grant MM-7.
} 


$$
\begin{array}{cc}
\dot{x}=f(t, x, Q x(t)) & \text { for } t \neq t_{i}, t \in[0, T] \\
\left.\Delta x\right|_{t=t_{i}}=I_{i}\left(x\left(t_{i}-0\right)\right) & \\
x(t)=\varphi(t) & \text { for } t \in[-h, 0],
\end{array}
$$

where $\quad x=\left(x_{1}, x_{2}, \ldots, x_{n}\right), \quad f:[0, T] \times \mathbb{R}^{n} \times \mathbb{R}^{n} \rightarrow \mathbb{R}^{n}, \quad f=\left(f_{1}, f_{2}, \ldots, f_{n}\right), \quad Q x=\left(Q_{1} x, Q_{2} x, \ldots\right.$, $\left.Q_{n} x\right), Q_{j} x(t)=\int_{t-h}^{t} k_{j}(t, s) x_{j}(s) d s, k_{j}:[0, T] \times[-h, T] \rightarrow[0, \infty), \varphi:[-h, 0] \rightarrow \mathbb{R}^{n}, \varphi=\left(\varphi_{1}, \varphi_{2}\right.$, $\left.\ldots, \varphi_{n}\right), h=$ const $>0,0<t_{1}<t_{2}<\ldots<t_{p}<T,\left.\Delta x\right|_{t=t_{i}}=x\left(t_{i}+0\right)-x\left(t_{i}-0\right), I_{i}: \mathbb{R}^{n} \rightarrow \mathbb{R}^{n}$, $I_{i}=\left(I_{i 1}, I_{i 2}, \ldots, I_{i n}\right)$.

With any integer $j=1, \ldots, n$, we associate two nonnegative integers $p_{j}$ and $q_{j}$ such that $p_{j}+q_{j}=n-1$ and introduce the notation

$$
\left(x_{j},[x]_{p_{j}},[y]_{q_{j}}\right)=\left\{\begin{array}{cc}
\left(x_{1}, x_{2}, \ldots, x_{p_{j}+1}, y_{p_{j}+2}, \ldots, y_{n}\right) & \text { for } p_{j} \geq j \\
\left(x_{1}, \ldots, x_{p_{j}}, y_{p_{j}+1}, \ldots, y_{j-1}, x_{j}, y_{j+1}, \ldots, y_{n}\right) & p_{j}<j .
\end{array}\right.
$$

With the notation introduced, the initial value problem (1) can be written down in the form

$$
\begin{gathered}
\dot{x}_{j}=f_{j}\left(t, x_{j},[x]_{p_{j}},[x]_{q_{j}}, Q_{j} x(t),[Q x(t)]_{p_{j}},[Q x(t)]_{q_{j}}\right) \text { for } t \neq t_{i}, t \in[0, T] \\
\left.\Delta x_{j}\right|_{t=t_{i}}=I_{i j}\left(x_{j}\left(t_{i}\right),\left[x\left(t_{i}\right)\right]_{p_{j}}\left[x\left(t_{i}\right)\right]_{q_{j}}\right) \\
x_{j}(t)=\varphi_{j}(t) \text { for } t \in[-h, 0], j=1, \ldots, n .
\end{gathered}
$$

Let $x, y \in \mathbb{R}^{n}, x=\left(x_{1}, x_{2}, \ldots, x_{n}\right), y=\left(y_{1}, y_{2}, \ldots, y_{n}\right)$. We shall say that $x \geq(\leq) y$ if for any $i=1, \ldots, n$, the inequality $x_{i} \geq(\leq) y_{i}$ holds.

Consider the set $G\left([a, b], \mathbb{R}^{n}\right)$ of all functions $u:[a, b] \rightarrow \mathbb{R}^{n}$ which are piecewise continuous with points of discontinuity of the first kind at the points $t_{i} \in(a, b)$, $u\left(t_{i}\right)=u\left(t_{i}-0\right)$ and the set $G^{1}\left([a, b], \mathbb{R}^{n}\right)$ of all functions $u \in G\left([a, b], \mathbb{R}^{n}\right)$ which are continuously differentiable for $t \neq t_{i}, t \in[a, b]$ and have continuous left derivatives at the points $t_{i} \in(a, b)$.

Definition 1: $\quad$ The couple of functions $v, w \in G\left([-h, T], \mathbb{R}^{n}\right), v, w \in G^{1}\left([0, T], \mathbb{R}^{n}\right)$, $v=\left(v_{1}, v_{2}, \ldots, v_{n}\right), w=\left(w_{1}, w_{2}, \ldots, w_{n}\right)$ is said to be a couple of lower and upper quasisolutions of the initial value problem (1) if the following inequalities hold.

$$
\begin{gathered}
\dot{v}_{j} \leq f_{j}\left(t, v_{j},[v]_{p_{j}},[w]_{q_{j}}, Q_{j} v,[Q v]_{p_{j}},[Q w]_{q_{j}}\right) \text { for } t \neq t_{i}, t \in[0, T] \\
\dot{w}_{j} \geq f_{j}\left(t, w_{j},[w]_{p_{j}}[v]_{q_{j}}, Q_{j} w,[Q w]_{p_{j}},[Q v]_{q_{j}}\right) \\
\left.\Delta v_{j}\right|_{t=t_{i}} \leq I_{i j}\left(v_{j}\left(t_{i}\right),\left[v\left(t_{i}\right)\right]_{p_{j}},\left[w\left(t_{i}\right)\right]_{q_{j}}\right)
\end{gathered}
$$




$$
\begin{gathered}
\left.\Delta w_{j}\right|_{t=t_{i}} \geq I_{i j}\left(w_{j}\left(t_{i}\right),\left[w\left(t_{i}\right)\right]_{p_{j}}\left[v\left(t_{i}\right)\right]_{q_{j}}\right) \\
v_{j}(t) \leq \varphi_{j}(t) \leq w_{j}(t) \text { for } t \in[-h, 0], j=1, \ldots, n .
\end{gathered}
$$

Definition 2: In the case when (1) is an initial value problem for a scalar impulsive integro-differential equation, i.e. $n=1$ and $p_{1}=q_{1}=0$, the couple of upper and lower quasisolutions of (1) are said to be upper and lower solutions of the same problem.

Definition 3: $\quad$ The couple of functions $v, w \in G\left([-h, T], \mathbb{R}^{n}\right), v, w \in G^{1}\left([0, T], \mathbb{R}^{n}\right)$ is said to be a couple of quasisolutions of the initial value problem (1) if (2), (3) and (4) hold only as equalities.

Definition 4: $\quad$ The couple of functions $v, w \in G\left([-h, T], \mathbb{R}^{n}\right), v, w \in G^{1}\left([0, T], \mathbb{R}^{n}\right)$ is said to be a couple of minimal and maximal quasisolutions of the initial value problem (1) if they are a couple of quasisolutions of the same problem and for any couple of quasisolutions of (1) $(u, z)$ the inequalities $v(t) \leq u(t) \leq w(t)$ and $v(t) \leq z(t) \leq w(t)$ hold for $t \in[-h, T]$.

Remark 1: $\quad$ Note that for the couple of minimal and maximal quasisolutions $(v, w)$ of (1) the inequality $v(t) \leq w(t)$ holds for $t \in[-h, T]$, while for an arbitrary couple of quasisolutions $(u, z)$ of (1) an analogous inequality may not be valid.

Remark 2: $\quad$ If for any $j=1, \ldots, n$, the equalities $p_{j}=n-1$ and $q_{j}=0$ hold and the couple of functions $(v, w)$ is a couple of quasisolutions of the initial value problem (1), then the functions $v(t)$ and $w(t)$ are two solutions of the same problem. If, in this case, problem (1) has a unique solution $u(t)$, then the couple of functions $(u, u)$ is a couple of minimal and maximal quasisolutions of (1).

For any couple of functions $v, w \in G\left([-h, T], \mathbb{R}^{n}\right), v, w \in G^{1}\left([0, T], \mathbb{R}^{n}\right)$ such that $v(t) \leq w(t)$ for $t \in[-h, T]$ define the set of functions

$$
S(v, w)=\left\{u \in G\left([-h, T], \mathbb{R}^{n}\right), u \in G^{1}\left([0, T], \mathbb{R}^{n}\right): v(t) \leq u(t) \leq w(t) \text { for } t \in[-h, T]\right\}
$$

\section{MAIN RESULTS}

Lemma 1: Let the following conditions hold:

1. The function $k \in C([0, T] \times[-h, T],[0, \infty))$.

2. The function $g \in G([-h, T], \mathbb{R}), g \in G^{1}\left([0, T], \mathbb{R}^{n}\right)$ satisfies the inequalities

$$
\dot{g}(t) \leq-M g(t)-N \int_{t-h}^{t} k(t, s) g(s) d s \text { for } t \neq t_{i}, t \in[0, T]
$$




$$
\begin{gathered}
\left.\Delta g\right|_{t=t_{i}} \leq-L_{i} g\left(t_{i}\right) \\
g(0) \leq g(t) \leq 0 \text { for } t \in[-h, 0]
\end{gathered}
$$

where $M, N, L_{i}(i=1, \ldots, p)$ are constants such that $M, N>0,0 \leq L_{i}<1$.

3. The inequality

$$
\left(M+N \kappa_{0} h\right) p \tau<(1-L)^{p}
$$

holds, where

$$
\begin{gathered}
\kappa_{0}=\max \{\kappa(t, s): t \in[0, T], s \in[-h, T]\}, \\
\tau=\max \left\{t_{1}, T-t_{p}, \max \left[t_{i+1}-t_{i}: i=1,2, \ldots, p-1\right]\right\} \\
L=\max \left\{L_{i}: i=1,2, \ldots, p\right\}
\end{gathered}
$$

Then $g(t) \leq 0$ for $t \in[-h, T]$.

Proof: Suppose that this is not true, i.e. that there exists a point $\xi \in[0, T]$ such that $g(\xi)>0$. The following three cases are possible:

Case 1: Let $g(0)=0$ and $g(t) \geq 0, g(t) \not \equiv 0$ for $t \in[0, b)$ where $b>0$ is a sufficiently small number. From inequality (7), it follows that $g(t) \equiv 0$ for $t \in[h, 0]$. Then by assumption there exist points $\xi_{1}, \xi_{2} \in[0, T], \xi_{1}<\xi_{2}$, such that $g(t)=0$ for $t \in\left[-h, \xi_{1}\right]$ and $g(t)>0$ for $t \in\left(\xi_{1}, \xi_{2}\right]$. From inequality (5), it follows that $\dot{g}(t) \leq 0$ for $t \in\left[\xi_{1}, \xi_{2}\right] \cap\left[\xi_{1}, \xi_{1}+h\right], t \neq t_{i}$, which together with inequality (6) shows that the function $g(t)$ is monotone nonincreasing in the interval $\left[\xi_{1}, \xi_{2}\right] \cap\left[\xi_{1}, \xi_{1}+h\right]$, i.e. $g(t) \leq g\left(\xi_{1}\right)=0$ for $t \in\left[\xi_{1}, \xi_{2}\right] \cap\left[\xi_{1}, \xi_{1}+h\right]$. The last inequality contradicts the choice of points $\xi_{1}$ and $\xi_{2}$.

Case 2: Let $g(0)<0$. By assumption and inequality (7) there exists a point $\eta \in(0, T], \eta \neq t_{i}(i=1, \ldots, p)$, such that $g(t) \leq 0$ for $t \in[-h, \eta), g(\eta)=0$ and $g(t)>0$ for $t \in(\eta, \eta+\epsilon)$ where $\epsilon>0$ is a sufficiently small number. Introduce the notation $\inf \{g(t): t \in[-h, \eta]\}=-\lambda, \lambda=$ const $>0$. Then there are two possibilities:

Case 2.1: Let a point $\rho \in[0, \eta]$ exist, $\rho \neq t_{i}(i=1, \ldots, p)$ such that $g(\rho)=-\lambda$. For the sake of definiteness, let $\rho \in\left(t_{k}, t_{k+1}\right]$ and $\eta \in\left(t_{k+m}, t_{k+m+1}\right], m \geq 0$. Choose a point $\eta_{1} \in\left(t_{k+m}, t_{k+m+1}\right], \eta_{1}<\eta$ such that $g\left(\eta_{1}\right)>0$. By the mean value theorem, the following equations are valid.

$$
g\left(\eta_{1}\right)-g\left(t_{k+m}+0\right)=\dot{g}\left(\xi_{m}\right)\left(\eta_{1}-t_{k+m}\right)
$$




$$
\begin{aligned}
& g\left(t_{k+m}-0\right)-g\left(t_{k+m-1}+0\right)=\dot{g}\left(\xi_{m-1}\right)\left(t_{k+m}-t_{k+m-1}\right) \\
& \begin{array}{lllllll}
\ldots & \ldots & \ldots & \ldots & \ldots & \ldots & \ldots
\end{array} \\
& g\left(t_{k+1}-0\right)-g(\rho)=\dot{g}\left(\xi_{0}\right)\left(t_{k+1}-\rho\right)
\end{aligned}
$$

where $\xi_{0} \in\left(\rho, t_{k+1}\right), \xi_{m} \in\left(t_{k+m}, \eta_{1}\right), \xi_{i} \in\left(t_{k+i}, t_{k+i+1}\right), i=1, \ldots, m-1$.

From (6) and (9) we obtain the inequalities

$$
\begin{gathered}
g\left(\eta_{1}\right)-\left(1-L_{k+m}\right) g\left(t_{k+m}\right) \leq \dot{g}\left(\xi_{m}\right) \tau \\
g\left(t_{k+m}\right)-\left(1-L_{k+m-1}\right) g\left(t_{k+m-1}\right) \leq \dot{g}\left(\xi_{m-1}\right) \tau \\
\ldots \quad \ldots \quad \ldots \quad \ldots l l l l l l \\
g\left(t_{k+1}\right)-g(\rho) \leq \dot{g}\left(\xi_{0}\right) \tau .
\end{gathered}
$$

From inequalities (10), by means of elementary transformations, we obtain the inequalities

$$
\begin{gathered}
g\left(\eta_{1}\right)-\left(1-L_{k+1}\right)\left(1-L_{k+2}\right) \ldots\left(1-L_{k+m}\right) g(\rho) \\
\leq\left[\dot{g}\left(\xi_{m}\right)-\left(1-L_{k+m}\right) \dot{g}\left(\xi_{m-1}\right)+\ldots+\right. \\
\left.\left(1-L_{\kappa+m}\right)\left(1-L_{\kappa+m-1}\right) \ldots\left(1-L_{\kappa+1}\right) \dot{g}\left(\xi_{0}\right)\right] \tau
\end{gathered}
$$

Inequalities (6) and (11) and the choice of the points $\rho$ and $\eta_{1}$ imply the inequality

$$
(1-L)^{m} \lambda<\left[1+\left(1-L_{\kappa+m}\right)+\ldots+\left(1-L_{\kappa+m}\right)\left(1-L_{\kappa+m-1}\right) \ldots\left(1-L_{\kappa+1}\right)\right]\left(M+N \kappa_{0} h\right) \tau \lambda
$$

or

$$
1<\frac{\left(M+N \kappa_{0} h\right)}{(1-L)^{p}} p \tau .
$$

Inequality (12) contradicts inequality (8).

Case 2.2: Let a point $t_{\kappa} \in[0, \eta)$ exist such that $g\left(t_{\kappa}+0\right)<g(t)$ for $t \in[0, \eta)$, i.e. $g\left(t_{\kappa}+0\right)=-\lambda$. By arguments analogous to those in Case 2.1, where $\rho=t_{\kappa}+0$, we again obtain a contradiction.

Case 3: Let $g(0)=0$ and $g(t) \leq 0, g(t) \not \equiv 0$ for $t \in(0, b]$ where $b>0$ is a sufficiently small number. By arguments analogous to those in Case 2 we obtain a contradiction.

This completes the proof of Lemma 1 . 
Theorem 1: $\quad$ Let the following conditions hold:

1. The couple of functions $v, w \in G\left([-h, T], \mathbb{R}^{n}\right), v, w \in G^{\mathbf{1}}\left([0, T], \mathbb{R}^{n}\right)$ is a couple of lower and upper quasisolutions of the initial value problem (1) and satisfies the inequalities $v(t) \leq w(t)$ for $t \in[-h, T]$ and $v(0)-\varphi(0) \leq v(t)-\varphi(t), w(0)-\varphi(0) \geq w(t)-\varphi(t)$ for $t \in[h, 0]$.

2. The functions $\kappa_{j} \in C([0, T] \times[-h, T],[0, \infty)), j=1, \ldots, n$.

3. The function $f \in C\left([0, T] \times \mathbb{R}^{n} \times \mathbb{R}^{n}, \mathbb{R}^{n}\right), f=\left(f_{1}, f_{2}, \ldots, f_{n}\right), f_{j}(t, x, y)=f_{j}\left(t, x_{j}\right.$, $[x]_{p_{j}},[x]_{q_{j}}, y_{j},[y]_{p_{j}},[y]_{q_{j}}$ ) is monotone nondecreasing with respect to $[x]_{p_{j}}$ and $[y]_{p_{j}}$ and monotone nonincreasing with respect to $[x]_{q_{j}}$ and $[y]_{q_{j}}$ and for $x, y \in S(v, w)$, $y(t) \leq x(t)$ satisfies the inequalities

$$
\begin{gathered}
f_{j}\left(t, x_{j},[x]_{p_{j}}[x]_{q_{j}}, Q_{j} x,[Q x]_{p_{j}},[Q x]_{q_{j}}\right) \\
-f_{j}\left(t, y_{j},[x]_{p_{j}}[x]_{q_{j}}, Q_{j} y,[Q x]_{p_{j}},[Q x]_{q_{j}}\right) \\
\geq-M_{j}\left(x y_{j}-y_{j}\right)-N_{j}\left(Q_{j} x-Q_{j} y\right), j=1, \ldots, n,
\end{gathered}
$$

where $M_{j}, N_{j}(j=1, \ldots, n)$ are positive constants.

4. The functions $I_{i} \in C\left(\mathbb{R}^{n}, \mathbb{R}^{n}\right), I_{i}=\left(I_{i 1}, I_{i 2}, \ldots, I_{i n}\right),(i=1, \ldots, p), I_{i j}(x)=I_{i j}\left(x_{j},[x]_{p_{j}}\right.$, $[x]_{q_{j}}$ ) are monotone nondecreasing with respect to $[x]_{p_{j}}$ and monotone nonincreasing with respect to $[x]_{q_{j}}$ and for $x, y \in S(v, w), y\left(t_{i}\right) \leq x\left(t_{i}\right)$ satisfy the inequalities

$$
\begin{gathered}
I_{i j}\left(x_{j}\left(t_{i}\right),\left[x\left(t_{i}\right)\right]_{p_{j}}\left[x\left(t_{i}\right)\right]_{q_{j}}\right)-I_{i j}\left(y_{j}\left(t_{i}\right),\left[x\left(t_{i}\right)\right]_{p_{j}},\left[x\left(t_{i}\right)\right]_{q_{j}}\right) \\
\geq-L_{i j}\left(x_{j}\left(t_{i}\right)-y_{j}\left(t_{i}\right)\right), j=1, \ldots, n, i=1, \ldots, p
\end{gathered}
$$

where $L_{i j}(i=1, \ldots, p, j=1, \ldots, n)$ are nonnegative constants, $L_{i j}<1$.

5. The inequalities

$$
\left(M_{j}+N_{j} \kappa_{0 j} h\right) \tau p \leq\left(1-L_{i}\right)^{p}, j=1, \ldots, n
$$

hold, where

$$
\begin{gathered}
\kappa_{0 j}=\max \left(\kappa_{j}(t, s): t \in[0, T], s \in[-h, T]\right\}, \\
\tau=\max \left\{t_{1}, T-t_{p}, \max \left[t_{i+1}-t_{i}: i=1,2, \ldots, p-1\right]\right\}, \\
L_{i}=\max \left\{L_{i j}: i=1,2, \ldots, p\right\} .
\end{gathered}
$$

Then there exist two monotone sequences of functions $\left\{v^{(\kappa)}(t)\right\}_{0}^{\infty}$ and $\left\{w^{(\kappa)}(t)\right\}_{0}^{\infty}$, $v^{(0)}(t) \equiv v(t), w^{(0)}(t) \equiv w(t)$ which are uniformly convergent in the interval $[-h, T]$ and their limits $\bar{v}(t)=\lim _{\kappa \rightarrow \infty} v^{(\kappa)}(t)$ and $\bar{w}(t)=\lim _{\kappa \rightarrow \infty} w^{(\kappa)}(t)$ are a couple of minimal and maximal 
quasisolutions of the initial value problem (1). Moreover, if $u(t)$ is any solution of the initial value problem (1) such that $u \in S(v, w)$, then the inequalities $\bar{v}(t) \leq u(t) \leq \bar{w}(t)$ hold for $t \in[-h, T]$.

Proof: Fix two functions $\eta, \mu \in S(v, w), \quad \eta\left(\eta_{1}, \eta_{2}, \ldots, \eta_{n}\right), \quad \mu=\left(\mu_{1}, \mu_{2}, \ldots, \mu_{n}\right)$. Consider the initial value problems for the linear impulsive integro-differential equations

and

$$
\begin{gathered}
\dot{x}_{j}+M_{j} x_{j}(t)+N_{j} \int_{t-h}^{t} \kappa_{j}(t, s) x_{j}(s) d s=\sigma_{j}(t, \eta, \mu) \text { for } t \neq t_{i}, t \in[0, T] \\
\left.\Delta x_{j}\right|_{t=t_{i}}=-L_{i j} x_{j}\left(t_{i}\right)+\gamma_{i j}(\eta, \mu) \\
x_{j}(t)=\varphi_{j}(t) \text { for } t \in[-h, 0]
\end{gathered}
$$

where

$$
\begin{aligned}
& \sigma_{j}(t, \eta, \mu)=f_{j}\left(t, \eta_{j},[\eta(t)]_{p_{j}},[\mu(t)]_{q_{j}}, Q_{j} \eta(t),[Q \eta(t)]_{p_{j}},[q \mu(t)]_{q_{j}}\right) \\
& \quad+M_{j} \eta_{j}(t)+N_{j} Q_{j} \eta(t), \\
& \gamma_{i j}(\eta, \mu)=I_{i j}\left(\eta_{j}\left(t_{i}\right),\left[\eta\left(t_{i}\right)\right]_{p_{j}}\left[\mu\left(t_{i}\right)\right]_{q_{j}}\right)+L_{i j} \eta_{j}\left(t_{i}\right), j=1, \ldots, n .
\end{aligned}
$$

The initial value problem (13)-(15) has a unique solution for any fixed couple of functions $\eta, \mu \in S(v, w)$.

Define the map $A: S(v, w) \times S(v, w) \rightarrow S(v, w)$ by the equality $A(\eta, \mu)=x$, where $x=\left(x_{1}, x_{2}, \ldots, x_{n}\right)$ and $x_{j}(t)$ is the unique solution of the initial value problem (13)-(15) for the couple of functions $\eta, \mu \in S(v, w)$.

We shall prove that $v \leq A(v, w)$. Introduce the notations $x^{(1)}=A(v, w), g=v-x^{(1)}$, $g=\left(g_{1}, g_{2}, \ldots, g_{n}\right)$. Then the following inequalities hold:

$$
\begin{aligned}
& \dot{g}_{j}(t)=\dot{v}-x^{(1)} \leq f_{j}\left(t, v_{j},[v]_{p_{j}},[w]_{q_{j}}, Q_{j} v,[Q v]_{p_{j}},[Q w]_{q_{j}}\right) \\
& +M_{j} x_{j}^{(1)}+N_{j} Q_{j} x^{(1)}-\sigma_{j}(t, v, w) \\
& =-M_{j} g_{j}(t)-N_{j} \int_{t-h}^{t} \kappa_{j}(t, s) g_{j}(s) d s \text { for } t \neq t_{i}, t \in[0, T], \\
& \left.\Delta g_{j}\right|_{t=t_{i}} \leq I_{i}\left(v_{j}\left(t_{i}\right),\left[v\left(t_{i}\right)\right]_{p_{j}}\left[w\left(t_{i}\right)\right]_{q_{j}}\right)+L_{i j} x_{j}^{(1)}\left(t_{i}\right)-\gamma_{i j}(v, w) \\
& =-L_{i j} g_{j}\left(t_{i}\right) \text {, } \\
& g_{j}(0) \leq g_{j}(t) \leq 0 \text { for } t \in[-h, 0], j=1, \ldots, n \text {. }
\end{aligned}
$$


By Lemma 1 , the functions $g_{j}(t), j=1, \ldots, n$ are nonpositive, i.e. $v \leq A(v, w)$. In an analogous way it is proved that $w \geq A(v, w)$.

Let $\eta, \mu \in S(v, w)$ be such that $\eta(t) \leq \mu(t)$ for $t \in[-h, T]$. Set $x^{(1)}=A(\eta, \mu)$, $x^{(2)}=A(\mu, \eta), g=x^{(1)}-x^{(2)}, g=\left(g_{1}, t_{2}, \ldots, g_{n}\right)$. By Lemma 1 the functions $g_{j}(t), j=1, \ldots, n$, are nonpositive, i.e. $A(\eta, \mu) \leq A(\mu, \eta)$.

Define the sequences of functions $\left\{v^{(\kappa)}(t)\right\}_{0}^{\infty}$ and $\left\{w^{(\kappa)}(t)\right\}_{0}^{\infty}$ by the equations

$$
\begin{gathered}
v^{(0)}(t) \equiv v(t), \quad w^{(0)}(t) \equiv w(t) \\
v^{(\kappa+1)}(t)=A\left(v^{(\kappa)}, w^{(\kappa)}\right), \quad w^{(\kappa+1)}(t)=A\left(w^{(\kappa)}, v^{(\kappa)}\right) .
\end{gathered}
$$

The functions $v^{(\kappa)}(t)$ and $w^{(\kappa)}(t)$ for $t \in[-h, T]$ and $\kappa \geq 0$ satisfy the inequalities

$$
v^{(0)}(t) \leq v^{(1)}(t) \leq \ldots \leq v^{(\kappa)}(t) \leq \ldots \leq w^{(\kappa)}(t) \leq \ldots \leq w^{(1)}(t) \leq w^{(0)}(t) .
$$

Hence the sequences of functions $\left\{v^{(\kappa)}(t)\right\}_{0}^{\infty}$ and $\left\{w^{(\kappa)}(t)\right\}_{0}^{\infty}$ are uniformly convergent for $t \in[-h, T]$. Introduce the notation $\bar{v}(t)=\lim _{\kappa \rightarrow \infty} v^{(\kappa)} t$ and $\bar{w}(t)=\lim _{\kappa \rightarrow \infty} w^{(\kappa)}(t)$. We shall show that the couple of functions $(\bar{v}, \bar{w})$ is a couple of minimal and maximal quasisolutions of the initial value problem (1). From the definitions of the functions $v^{(\kappa)}(t)$ and $w^{(\kappa)}(t)$, it follows that these functions satisfy the initial value problem

$$
\begin{gathered}
\dot{v}_{j}^{(\kappa+1)}+M_{j} v_{j}^{(\kappa+1)}+N_{j} Q_{j} v^{(\kappa+1)}=\sigma_{j}\left(t, v^{(\kappa)}, w^{(\kappa)}\right) \text { for } t \neq t_{i}, t \in[0, T] \\
\dot{w}_{j}^{(\kappa+1)}+M_{j} w_{j}^{(\kappa+1)}+N_{j} Q_{j} w^{(\kappa+1)}=\sigma_{j}\left(t, w^{(\kappa)}, v^{(\kappa)}\right), \\
\left.\Delta v_{j}^{(\kappa+1)}\right|_{t=t_{i}}=-L_{i j} v_{j}^{(\kappa+1)}\left(t_{i}\right)+\gamma_{i j}\left(v^{(\kappa)}, w^{(\kappa)}\right) \\
\left.\Delta w_{j}^{(\kappa+1)}\right|_{t=t_{i}}=-L_{i j} w_{j}^{(\kappa+1)}\left(t_{i}\right)+\gamma_{i j}\left(w^{(\kappa)}, v^{(\kappa)}\right), \\
v_{j}^{(\kappa+1)}(t)=w_{j}^{(\kappa+1)}(t)=\varphi_{j}(t) \text { for } t \in[-h, 0], j=1, \ldots, n .
\end{gathered}
$$

We pass to the limit in equations (18)-(20) and obtain that the functions $\bar{v}(t)$ and $\bar{w}(t)$ are a couple of quasisolutions of the initial value problem (1). From inequalities (17) it follows that the inequality $\bar{v}(t) \leq \bar{w}(t)$ holds for $t \in[-h, T]$.

Let $\zeta, z \in S(v, w)$ be a couple of quasisolutions of problem (1). From inequalities (17) it follows that there exists an integer $\kappa \geq 1$ such that $v^{(\kappa-1)}(t) \leq \zeta(t) \leq w^{(\kappa-1)}(t)$ and $v^{(\kappa-1)}(t) \leq z(t) \leq w^{(\kappa-1)}(t)$ for $t \in[-h, T]$. Introduce the notation $g(t)=v^{(\kappa)}(t)-\zeta(t)$, $g=\left(g_{1}, g_{2}, \ldots, g_{n}\right)$. By Lemma 1 , the inequality $g_{j}(t) \leq 0$ holds for $t \in[-h, T], j=1, \ldots, n$, i.e. $v^{(\kappa)}(t) \leq \zeta(t)$. 
In an analogous way, it is proved that the inequalities $\zeta(t) \leq w^{(\kappa)}(t)$ and $v^{(\kappa)}(t) \leq z(t) \leq w^{(\kappa)}(t)$ hold for $t \in[-h, T]$, which shows that the couple of functions $(\bar{v}, \bar{w})$ is a couple of minimal and maximal quasisolutions of the initial value problem (1).

Let $u(t)$ be a solution of (1) such that $u \in S(v, w)$. Consider the couple of functions $(u, u)$ which is a couple of quasisolutions of problem (1). By what was proved above, the inequalities $\bar{v}(t) \leq u(t) \leq \bar{w}(t)$ hold for $t \in[-h, T]$.

This completes the proof of Theorem 1 .

In the case when (1) - is an initial value problem for a scalar impulsive integrodifferential equation, the following theorem is valid.

Theorem 2: $\quad$ Let the following conditions hold:

(1) The functions $v, w \in G([-h, T], \mathbb{R}), v, w \in G^{1}([0, T], \mathbb{R})$ are a couple of lower and upper solutions of the initial value problem (1) and satisfy the inequalities $v(t) \leq w(t)$ for $t \in[-h, T]$ and $v(0)-\varphi(0) \leq v(t)-\varphi(t), w(0)-\varphi(0) \geq w(t)-\varphi(t)$ for $t \in[-h, 0]$.

(2) The function $\kappa(t, s) \in C([0, T] \times[-h, T],[0, \infty))$.

(3) The function $f \in C([0, T] \times \mathbb{R} \times \mathbb{R}, \mathbb{R})$ satisfies for $x, y \in S(v, w), y(t) \leq x(t)$ the inequality

$$
\begin{gathered}
f\left(t, x(t), \int_{t-h}^{t} \kappa(t, s) x(s) d s\right)-f\left(t, y(t), \int_{t-h}^{t} \kappa(t, s) y(s) d s\right) \\
\geq-M(x(t)-y(t))-N \int_{t-h}^{t} \kappa(t, s)(x(s)-y(s)) d s
\end{gathered}
$$

where $M$ and $N$ are positive constants.

(4) The function $I_{i} \in C(\mathbb{R}, \mathbb{R}) \quad(i=1, \ldots, p)$ satisfies for $x, y \in S(v, w), y\left(t_{i}\right) \leq x\left(t_{i}\right)$ the inequality $I_{i}\left(x\left(t_{i}\right)\right)-I_{i}\left(y\left(t_{i}\right)\right) \geq-L_{i}\left(x\left(t_{i}\right)-y\left(t_{i}\right)\right), i=1, \ldots, p$ where $L_{i}(i=1, \ldots, p)$ are nonnegative constants such that $L_{i}<1$.

(5) The inequality

$$
\left(M+N \kappa_{0} h\right) p \tau<(1-L)^{p}
$$

holds, where

$$
\begin{gathered}
\kappa_{0}=\max \{\kappa(t, s): t \in[0, T], s \in[-h, T]\}, \\
\tau=\max \left\{t_{1}, T-t_{p}, \max \left[t_{i+1}-t_{i}: i=1,2, \ldots, p-1\right]\right\} \\
L=\max \left\{L_{i}: i=1,2, \ldots, n\right\} .
\end{gathered}
$$

Then there exist two sequences of functions $\left\{v^{(\kappa)}(t)\right\}_{0}^{\infty}$ and $\left\{w^{(\kappa)}(t)\right\}_{0}^{\infty}$ which are uniformly 
convergent in the interval $[-h, T]$ and their limits $\bar{v}(t)=\lim _{\kappa \rightarrow \infty} v^{(\kappa)}(t)$ and $\bar{w}(t)=\lim _{\kappa \rightarrow \infty} w^{(\kappa)}(t)$ are a couple of minimal and maximal solutions of the initial value problem (1).

The proof of Theorem 2 is analogous to the proof of Theorem 1.

\section{REFERENCES}

[1] Deimling, K. and Lakshmikantham, V., Quasisolutions and their role in the qualitative theory of differential equations, Nonlinear Anal., 4, 657-663, 1980.

[2] Ladde, G.S., Lakshmikantham, V. and Vatsala, A.S., Monotone Iterative Techniques in Nonlinear Differential Equations, Pitman, Belmont, CA, 1985.

[3] Lakshmikantham, V., Monotone iterative technology for nonlinear differential equations, Coll. Math. Soc. Janos Bolyai, 47, Diff. Eq., Szeged, 633-647, 1984.

[4] Lakshmikantham, V. and Leela, S., Existence and monotone method for periodic solutions of first order differential equations, J. Math. Anal. Appl., 91, 237-243, 1983.

[5] Lakshmikantham, V. and Leela, S., Remarks on first and second order periodic boundary value problems, Nonlinear Anal., 8, 281-287, 1984.

[6] Lakshmikantham, V., Leela, S. and Oguztoreli, M.N., Quasi-solutions, vector Lyapunov functions and monotone method, IEEE Trans. Automat. Control, 26, 1149-1153, 1981.

[7] Lakshmikantham, V., Leela, S. and Vatsala, A.S., Method of quasi upper and lower solutions in abstract cones, Nonlinear Anal., 6, 833-838, 1982.

[8] Lakshmikantham, V. and Vatsala, A.S., Quasisolutions and monotone method for systems of nonlinear boundary value problems, J. Math. Anal. Appl., 79, 38-47, 1981. 


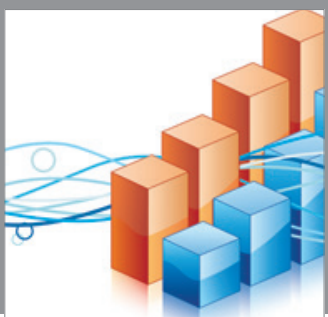

Advances in

Operations Research

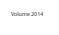

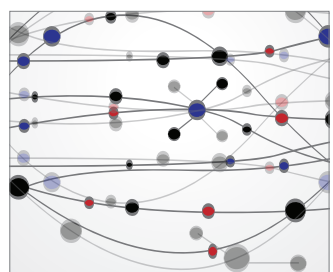

\section{The Scientific} World Journal
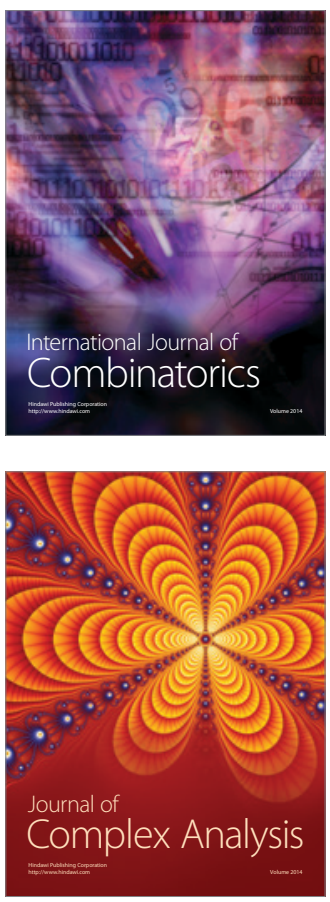

International Journal of

Mathematics and

Mathematical

Sciences
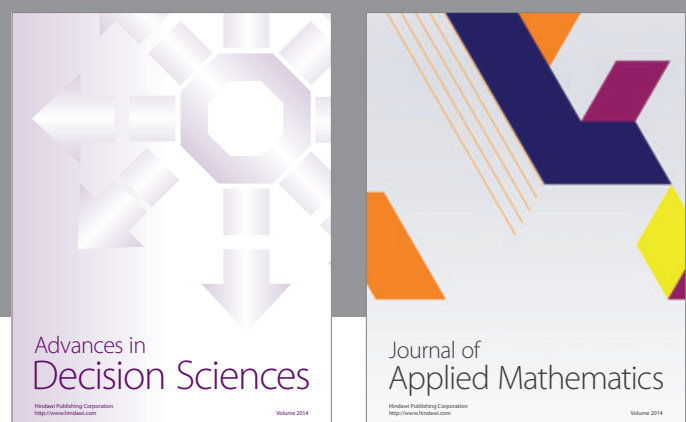

Journal of

Applied Mathematics
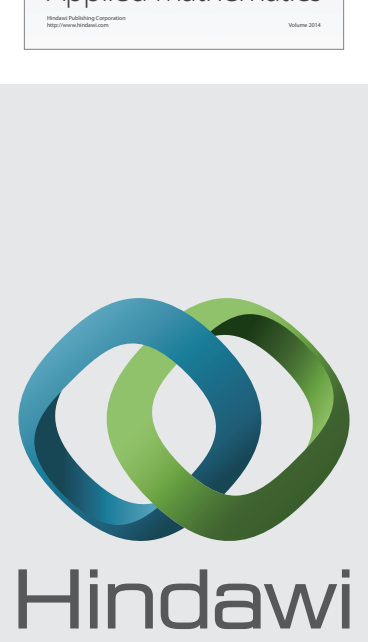

Submit your manuscripts at http://www.hindawi.com
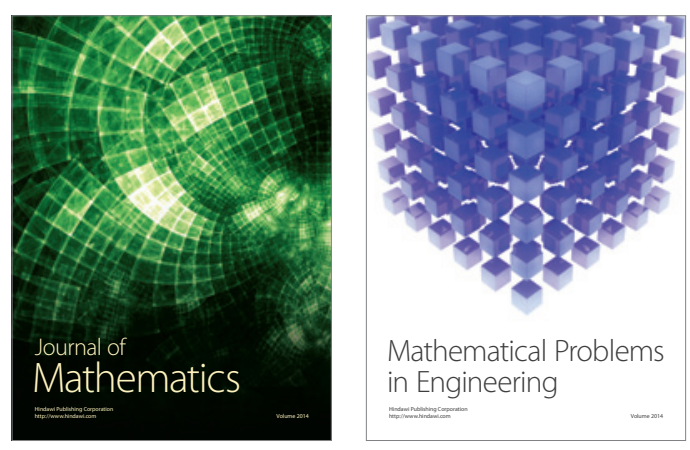

Mathematical Problems in Engineering
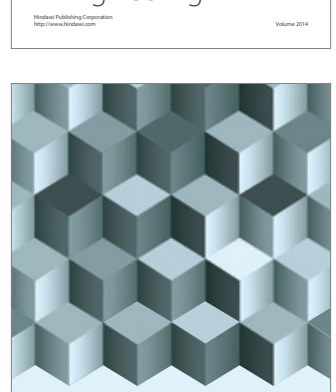

Journal of

Function Spaces
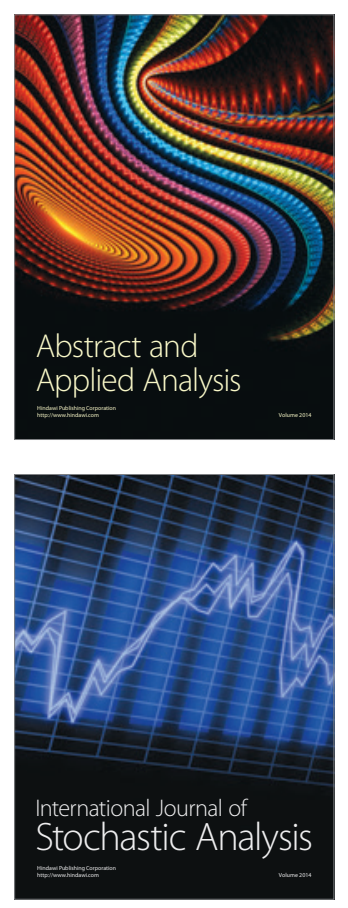

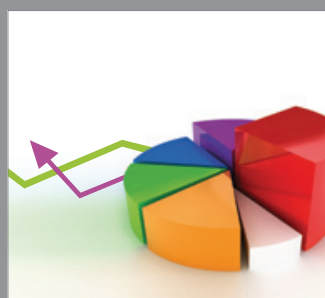

ournal of

Probability and Statistics

Promensencen
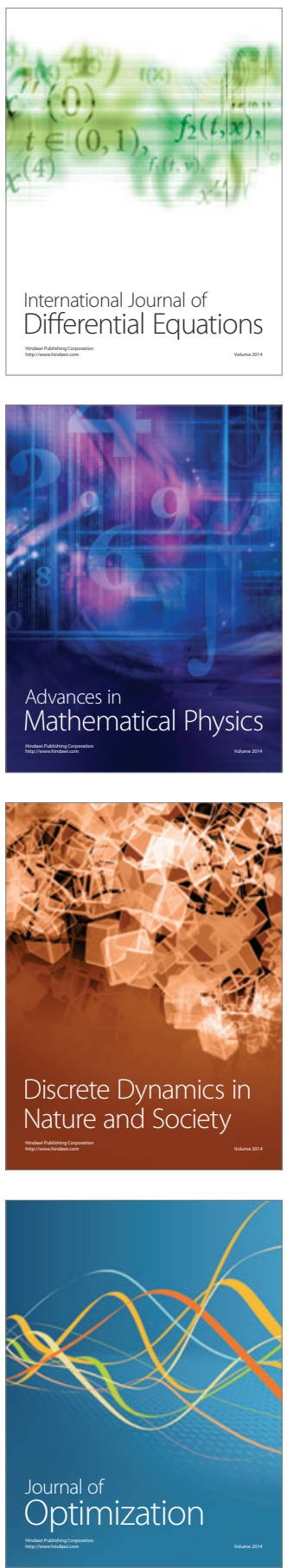